\title{
Política Pública, Autocontrol y Tendencia Intertemporal en el Control de la Obesidad y Sobrepeso
}

\author{
Política Pública, Autocontrole e Viés Intertemporal no Controle da \\ Obesidade e Sobrepeso
}
Public Policy, Self-Control and Intertemporal Trend in the Control of Obesity and Overweight

Politique Publique, Auto-Contrôle et Tendance Intertemporaine dans le Contrôle de l'Obésité et du Surpoids

Solon Bevilacqua ${ }^{1}$ e John Edwrd Neira Villena ${ }^{2}$

\footnotetext{
${ }^{1}$ Licenciado en Administración por la Universidade Federal do Rio Grande do Sul, Porto Alegre, RS, Brasil, Máster en Administración por la Universidade Federal de Uberlândia, Uberlândia, MG, Brasil, y doctorado en Psicología por la Pontifícia Universidade Católica de Goiás, Goiânia, GO, Brasil. En la actualidad es profesor de la Universidad Federal de Goiás, Catalão, GO, Brasil, donde coordina el Máster Profesional en Administración Pública y dirige el Grupo de Investigación M2C - Estudios de Mercado, Consumo y Salud.

E-mail: solbebv@gmail.com

${ }^{2}$ Licenciado en Ingeniería Mecánica por la Universidad Católica de Santa María, Arequipa - Perú, Máster en Ingeniería Mecánica y Doctor en Ciencia e Ingeniería de los Materiales por la Universidade Federal do Rio Grande do Norte, Natal, RN, Brasil, Actualmente es Gerente del Instituto SENAI de Innovación en Automatización en la Faculdade SENAI de Tecnologia Italo Bologna, Goiânia, GO, Brasil.
} 
Resumen

\section{Resumo}

Abstract
Una de las políticas públicas más importantes en pauta por la Organización Mundial de la Salud (OMS) es la que aborda la obesidad y el sobrepeso. En este artículo se desarrolló el autocontrol bajo el desafío de una dieta alimentar. Se investigo durante 4 meses, participantes interesados en perder peso, tal como un emprendedor que exige disciplina y autocontrol diario en su actividad laboral. La metodología adoptada utilizó tres variaciones de monitoreo: uso de adipómetro, control de medidas e Índice de Masa Corporal (IMC), en contraste con un grupo de control. Las descubiertas indican que las tres formas de monitoreo interfirieron poco en las prácticas saludables, pero el grupo que no fue monitoreado tuvo significativo aumento de peso. Fueron previstas contribuciones prácticas en el estudio, indicando que la mejor forma de sustentar el autocontrol en la práctica de una dieta es el monitoreo del peso hecho por terceros, independiente de cual control sea realizado.

Palabras Clave: Comportamiento; Autocontrol; Obesidad; Política Pública, Salud.

Uma das políticas públicas mais importantes para a Organização Mundial de Saúde (OMS) é a abordagem sobre obesidade e sobrepeso. Este artigo descreve o autocontrole como empreendimento de uma dieta alimentar. Foi investigado durante 4 meses participantes interessados em perder peso, como aquele empreendedor que exige disciplina e autocontrole diario em sua atividade laboral. A metodologia adotada utilizou três variações de monitoração: uso do adipômetro, controle de medidas e Índice de Massa Corporal (IMC), em contraste com um grupo de controle. Os achados da pesquisa indicaram que as três formas de monitoramento pouco influenciaram nas práticas saudáveis, mas o grupo que não foi monitorado teve aumento significativo de ganho de peso. Foram previstas contribuições práticas no estudo, indicando que a melhor forma de sustentar o autocontrole na prática de uma dieta é o monitoramento de peso feito por terceiros, independente da prática efetuada.

Palavras-Chave: Comportamento; Autocontrole; Obesidade; Política Publica, Saúd.

challenge of a diet. Participants interested in losing weight were investigated for 4 months, as well as a venture that requires discipline and daily self-control. The adopted methodology used three variations of monitoring: use of adipometer, control of measures and Body Mass Index (BMI), in contrast to a control group. The findings indicate that the three forms of monitoring had little influence on healthy practices, but that group that was not monitored had significant weight gain. Practical contributions were foreseen in the study, indicating that the best way to sustain self-control in the practice of a diet, is the monitoring of weight done by third parties, regardless of which control is performed.

Keywords: Behavior; Self-Control; Obesity; Public Policy; Health. 
Resumé L'une des politiques publiques les plus importantes à l'ordre du jour de l'Organisation mondiale de la santé (OMS) est celle qui traite de l'obésité et du surpoids. Dans cet article, la maitrise de soi a été développée sous le défi d'un régime. Les participants intéressés à perdre du poids ont été examinés pendant 4 mois, ainsi qu'une entreprise qui nécessite de la discipline et une maîtrise de soi au quotidien. La méthodologie adoptée a utilisé trois variantes de surveillance: l'utilisation d'un adipomètre, le contrôle des mesures et l'indice de masse corporelle (IMC), contrairement à un groupe témoin. Les résultats indiquent que les trois formes de surveillance ont eu peu d'influence sur les pratiques saines, mais que le groupe qui n'a pas été surveillé a eu un gain de poids significatif. Des contributions pratiques ont été prévues dans l'étude, indiquant que la meilleure façon de maintenir la maitrise de soi dans la pratique d'un régime alimentaire est la surveillance du poids effectuée par des tiers, quel que soit le contrôle effectué.

Mots-Clés: Comportement; Maîtrise de soi; Obésité; Politique publique; Santé. 


\section{Introdução}

Sobrepeso y obesidad ya fueron clasificados por la Organización Mundial de la Salud (OMS) como epidémicos, lo que sugiere algunas prácticas que contribuyan con políticas públicas eficaces. En ese sentido, esta investigación desarrolla de forma práctica una idea oriunda de la psicología económica, centrada en el autocontrol y combate al agotamiento del ego. Los resultados de la investigación indicaron que el monitoreo auxilia a perder de peso, mientras que la forma con que ese control es ejercido es irrelevante.

Se constata la importancia de estudios como este, cuando se nota el trabajo de la Organización Mundial de Salud (OMS) en inhibir las proporciones epidémicas del sobrepeso u obesidad al combatir la ingestión de alimentos no saludables y, en paralelo, incentivar la actividad física. Se trata de una actuación centrada en el proceso de selección de la población, ya que se trata de una epidemia ambiental y social (World Health Organization, 2017).

Superar el problema de la obesidad, emprender una dieta y ejercitarse incluye vencer una serie de desafíos de la vida moderna y, fundamentalmente, ejercitar el autocontrol. Así como una estructura muscular, el autocontrol demanda desarrollo y disciplina para que sea posible extraer de su estructura la respuesta esperada. De forma análoga a un músculo, que si trabajado, gana fuerza y resistencia. El autocontrol puede ser desarrollado (Selart e cols., 1997), ya sea para perseguir un objetivo, o sea para remover estímulos negativos, rutineros e inútiles (Thaler, 2008).

A partir del objetivo de desarrollar el autocontrol con el desafío de una dieta alimentar, se investigó durante 4 meses, participantes interesados en perder peso, tal como un emprendimiento que exige disciplina y autocontrol diario. Además de los resultados prácticos, se cree que la teoría relacionada pueda contribuir en gran parte para los estudios sobre seguridad alimentar y políticas públicas sobre la obesidad y sobrepeso, tal como contribuyó para la gran área de la sicología económica.

En este estudio fueron relacionadas tres hipótesis: el desarrollo de una dieta pierde fuerza a lo largo del proceso (depleción del ego) $\left(\mathrm{H}_{1}\right)$; el monitoreo a períodos fijos incrementa la taza de éxito $\left(\mathrm{H}_{2}\right)$; el modelo de monitoreo interfiere en el resultado esperado $\left(\mathrm{H}_{3}\right)$. El soporte teórico de esta investigación prevé que la selección a lo largo del tiempo sufre interferencias temporales y falla del compromiso, juntas, interfieren en el éxito del autocontrol. También se presume que las consecuencias postergadas son intangibles en virtud de la acción del comportamiento presente que es imperceptible. Ingerir una comida calórica, por ejemplo, causa una acción imperceptible en el aumento de peso futuro, mientras es perceptible el sacrificio a ser emprendido en el presente para no ingerirla (Smith, 2004, 2006). Se entiende también, a partir de la teoría presentada a continuación, que el autocontrol puede ser trabajado como una estructura muscular, por esfuerzos frecuentes, conforme estudios encontrados (Baumeister e cols., 1994; Gailliot, Baumeister, Dewall, e cols., 2007). 


\section{Autocontrol y Tendencia Intertemporal}

Los efectos temporales pueden entrar en proceso de interacción, alternándose o presentándose en secuencia. Para el mismo agente, la anomalía de miopía puede surgir para un consumo específico y, simultáneamente, para otro consumo, presentarse como hipermetropía. En este caso el descuento excesivo del presente se opone al futuro imaginado. Considerando el ejemplo de ahorrar, un consumidor puede decidir reducir sus gastos y guardar sus recursos (hipermetropía) envés de gastar hoy y despreocuparse con el futuro (miopía) (Selart e cols., 1997).

Cuando el agente no consigue actuar conforme planeado, presentando inconsistencia temporal, o sea, se propone a realizar algo, pero concluye otra acción. Se dice que hubo una fatiga del autocontrol (Hagger e cols., 2016). El autocontrol y su interacción con los efectos intangibles de la selección intertemporal son particularmente interesantes para los estudios de salud pública, por algunas cuestiones emergentes. A partir de los primeros estudios sobre autocontrol, ese estado visceral pasó a ser visto como una energía que es usada por los músculos, con stock limitado (Baumeister e cols., 1994). Los comportamientos de autocontrol son proyectados para maximizar los intereses de largo plazo del individuo (Mischel e cols., 1972). Es el ejercicio del control sobre el mí por mí, o sea, el autocontrol ocurre cuando una persona (u otro organismo) intenta mudar la manera como él o ella pensaría, sentiría o se comportaría.

Además de monitorear las prácticas en este trabajo, las descubiertas que envuelven el estudio del autocontrol sobre tendencia intertemporal determinan la práctica de la eliminación de opciones. De forma metafórica, sería como mantener alejada determinada tentación, que ofrece la oportunidad al agente, conforme prevista en la clásica Ilíada.

Homero describe Ulisses como un semidios atormentado por tentaciones humanas. El personaje es amarrado por la tripulación al mástil de su propio navío. Se buscaba la idea de no sucumbir al canto seductor de las sirenas y lograr continuar navegando. La práctica del autocontrol, en ese caso, fue un éxito a través de la eliminación de opciones. El comprometimiento fue la consecuencia del éxito de la estrategia (Thaler, 2008).

En la misma dirección de esa idea de promover control y facilitar algunas prácticas centradas en un objetivo definido, Richard Thaler fue agraciado con el Premio Nobel em 2017, de la teoría y práctica del empleo de nudges como política pública. Una de sus hipótesis relaciona el autocontrol como factor determinante de éxito.

El agotamiento del ego no significa que el individuo se quedó sin energía para el autocontrol, pero que estaría ocurriendo una práctica de conservación de energía para otras demandas. Y así como los músculos quedan más fuertes con el ejercicio, el autocontrol podría ser mejorado por esfuerzos frecuentes (Baumeister e cols., 2006). Además de la teoría propuesta, experimentos en forma de meta análisis fueran realizados recientemente corroborando con las descubiertas de dos décadas de investigación (Beames e cols., 2017; Friese e cols., 2017). El ejercicio frecuente de autocontrol seguido de la oportunidad de reposo y reposición total puede aumentar gradualmente la fuerza total del individuo para el autocontrol. En situación análoga, el esfuerzo puede ser utilizado a punto de fatiga, exigiendo del individuo una fase de recuperación muscular. También fue previsto a partir de los estudios relacionados, 
que el autocontrol puede ser preservado para un evento de explosión. Esa sería una situación atípica, estratégica y, digna de una superación para el individuo (Muraven \& Baumeister, 2000).

La pregunta relacionada a la fatiga del autocontrol también puede ser presentada de forma didáctica con el ejemplo de un corredor y sus límites. Por más que esté motivado, un corredor no puede pasar sus límites físicos, por lo tanto su autocontrol tendría una especie de pico inicial, perdiendo fuerza a medida que la condición física lo permitiera (Holton, 2009). En ese sentido, se instala la primera hipótesis de la investigación: el desempeño de una dieta pierde fuerza a lo largo del proceso (depleción del ego) $\left(\mathrm{H}_{1}\right)$

El autocontrole y los efectos intangibles de la selección intertemporal pueden ser esclarecidos a partir de ejemplos básicos como el éxito de una dieta, consumo de bebidas alcohólicas, ahorrar para una gran compra futura, compras por impulso, endeudamiento y otras metas, compromisos o progresos (Wänke, 2009), inclusive procrastinar y empeñarse en los estudios (Job e cols., 2015), no ejercitarse lo suficiente, arañar una erupción cutánea, roer uñas, fumar, envolverse en sexo inseguro, abusar de drogas, gastar excesivamente e así por delante (Ariely \& Wertenbroch, 2002). Tales ejemplos pueden ser explorados en políticas públicas como reducir del consumo de bebidas alcohólicas o tabaco en poblaciones específicas, a partir de prácticas como nudges que actúan en el sistema de autocontrol.

Para evitar tales conflictos, las personas frecuentemente se imponen una serie de impedimentos, como, por ejemplo, en un caso de dieta, dejar de ir a un restaurante que posee un menú rico en calorías o evitar comprar determinados alimentos super procesados. Aún no está claro en los estudios que documentaron el comportamiento de autocontrol si los intentos de imponer restricciones a si propio son exitosas (Ariely \& Wertenbroch, 2002), mientras que, se sabe que las personas son conscientes de su falta de autocontrol y que tienen dificultad de gerenciar opciones, lo que induce la procrastinación (O’Donoghue \& Rabin, 2001). Se observa que el sistema está en conflicto. Algo como si la razón luchase con las emociones. También es posible entender que el control puede ser ejercido mediante la colocación de metas constantes que aún pueden ser revistas periódicamente. De esa forma se instala la segunda hipótesis de la investigación: $\mathrm{H}_{2}$ : el monitoreo a períodos fijos incrementa la taza de éxito.

Escoger entre una rodaja de torta y una ensalada de frutas, en situación de estrés, también involucra una disputa entre el sistema dual. Personas que están cognitivamente ocupadas tienden a optar por dulces (Shiv \& Fedorikhin, 2010); usar leguaje sexista, hacer juicios superficiales (Kahneman, 2011). La cuestión reside en un hecho relacionado al esfuerzo y la atención: todo esfuerzo cognitivo, emocional o físico demanda una reserva compartida de energía mental.

En experimentos recientes, quedo evidente que el agotamiento del ego (ego depletion) impide a los participantes a realizar actividades que involucren concentración o fuerza física. Después de ejercer el autocontrol en una tarea, el individuo no se siente dispuesto a emprender esfuerzo en otra tarefa (Kahneman, 2011). El sistema nervioso consume más glucosa que otras partes del cuerpo, tornando la actividad mental alta en consumo de energía. Estando el individuo involucrado en una actividad de raciocinio cognitivo difícil u ocupado, el nivel de glucosa en la sangre cae (Gailliot, Baumeister, Dewall, e cols., 2007).

En la figura 1 se muestran los "nosotros" que presentan los componentes principales relacionados a las teorías actuales sobre selección intertemporal y autocontrol. En el modelo de la figura 1, el deseo y el objetivo de orden superior son parcialmente incompatibles y generan el conflicto [a] deseo-meta, que activa a [b] motivación del control. La motivación y la capacidad de 
control interactivamente determinan el esfuerzo de control potencial. El esfuerzo de control real invertido es determinado por varios moderadores, incluyendo la fuerza de deseo, habilidad percibida y objetivos oponentes. El esfuerzo real de control y la fuerza del deseo compiten para determinar una fuerza predominante, que, en última análisis, determina el comportamiento, desde que las restricciones de ejecución no lo impidan (Kotabe \& Hofmann, 2015).

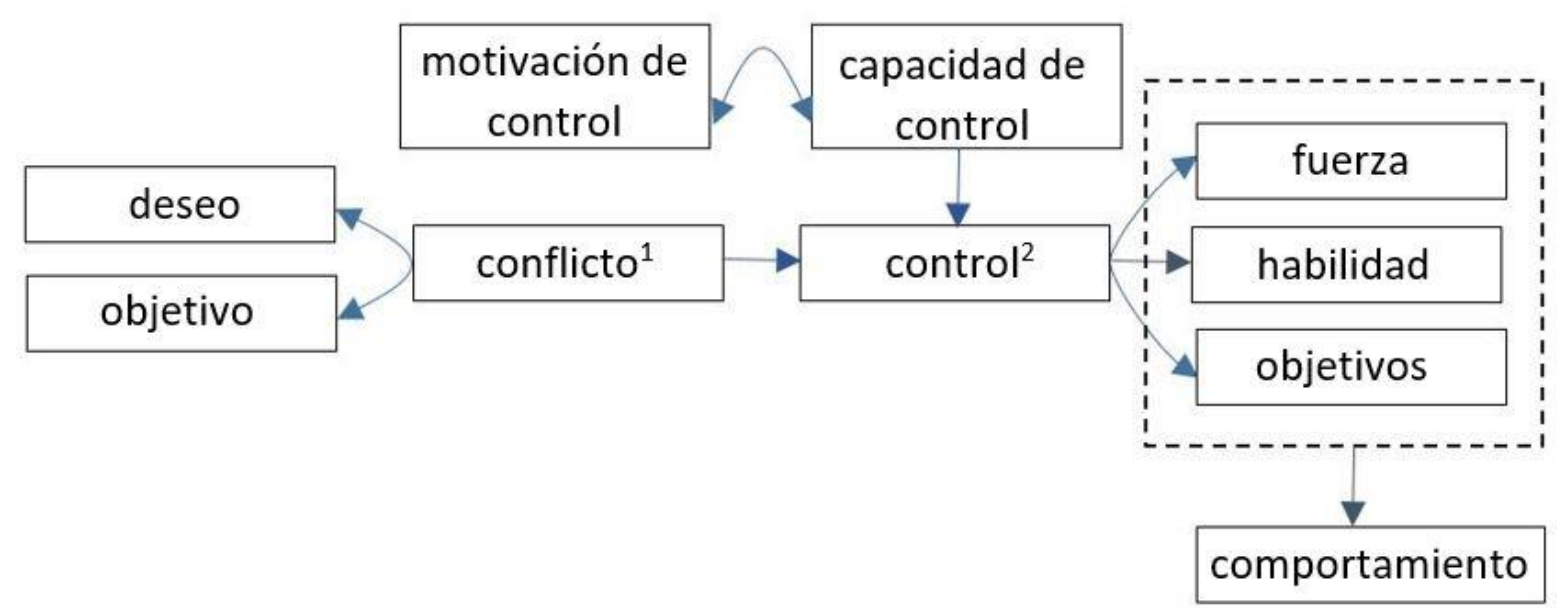

Figura 1. conexión entre las teorías de autocontrol. Goiânia, GO, 06 octubre de 2019

Fuente: Diagrama Adaptado de Autocontrol (Calder e cols., 2016; Laibson, 1996, 1997; O’Donoghue \& Rabin, 1992; Phelps \& Pollak, 1968)

En la figura 1 se contempla una iniciativa de crear un enlace entre las teorías de autocontrol. Ha sido esa la meta de investigadores de economía comportamental y sicología económica, que investigaron el autocontrol en varios niveles de complejidad (Kotabe \& Hofmann, 2015).

Las 'restricciones comportamentales', agentes importantes para la comprensión de por qué las personas no actúan con base al deseo, despertaron la atención de economistas comportamentales interesados en recrear ambientes de selección que expliquen el autocontrol (Thaler e cols., 2013; Thaler \& Sunstein, 2009).

\section{Material y Métodos}

Esta investigación fue aprobada por el comité de ética en investigación de la Universidade Federal de Goiás, según recomendaciones del Conselho Nacional de Pesquisa, con el registro 43341315.6.0000.5083. El período de colecta de datos corresponde al intervalo de marzo de 2018 a julio de 2018.

De forma aleatoria, 32 participantes fueron seleccionados y colocados en 3 grupos de investigación y 1 grupo de control, denominados [1] "IMC", [2] "peso", [3] "medidas" y [4] "control". Se registró 67,8\% de hombres en la muestra y 32,2\% de mujeres; universitarios en su 
totalidad, con predominio de jóvenes con edad media de 26,7 años (35,6\%). Se definió como criterio de inclusión el interés por perder peso y disponibilidad para participar voluntariamente durante 4 meses de acompañamiento semanal. Como criterio de exclusión, se consideró individuos inseridos en el emprendimiento de una dieta estructurada y las personas imposibilitadas de realizar el control a lo largo de los 4 meses de trabajo. Los individuos adeptos de una dieta estructurada podrían colocar la investigación en situación de descontrol. Dietas estructuradas demandan monitoreo de la taza de glucosa a partir de acompañamiento especializado. Bajos índices de glucosa en la sangre, ya indicaron depleción del ego e falencia del autocontrol (6).

La cuestión 'monitoreo de peso', uno de los niveles de variable operacional (aliada al IMC y medidas) es contestada por la comunidad especializada (nutricionistas, educadores, atletas...) ya que difícilmente refleja la realidad de la pérdida de peso (el individuo puede, por ejemplo, estar ganando masa magra y ganando peso). En virtud de esa cuestión, se nivelaron todas las medidas para la variación estadística del antes y después, envés de identificar pérdidas y ganancias en números absolutos.

Los grupos fueron definidos en función de orientación de nutricionistas, fue previsto a partir de los apuntes iniciales que no hay una manera segura de acompañar la pérdida de peso de un participante (el participante puede retener líquido; ganar masa magra; presentar otras características que promuevan el descontrol del estudio). La idea es crear soporte para la hipótesis 2, que sugiere la "variación" en los resultados obtenidos en función del método de medición (IMC, medidas, peso, entre otros).

El material usado fue un adipómetro, cintas métricas y balanzas digitales. Fue elaborada también una cabina-probador para registrar las medidas con privacidad.

Durante de 4 meses los participantes tuvieron sus medidas y peso registrados. Los procedimientos eran realizados en la cabina que preservaba la privacidad de los participantes. Los datos eran registrados en una planilla y presentados a los participantes como forma de registro e identificación posterior de la práctica del autocontrol.

Fueron realizadas pruebas para obtención de normalidad; pruebas para diferencias entre medias (prueba t) y análisis de variancia (Anova). Los softwares utilizados fueron el G*Power $3.1 \AA$ para definir la muestra; Systat $13 \AA$ para análisis de las medias y Excel ${ }^{\circledR}$ para análisis primarias.

\section{Resultados}

Las primeras análisis objetivan probar $\mathrm{H}_{1}$, si el desempeño de una dieta pierde fuerza a lo largo del proceso (depleción del ego); $\mathrm{H}_{2}$, si el monitoreo a períodos fijos incrementa la taza de éxito y $\mathrm{H}_{3}$, si el tipo de control ejercido interfiere en el resultado $\left(\mathrm{H}_{0}: \mu_{1}-\mu_{2=c e r o}\right)$. La prueba de Shapiro-Wilk evidenció normalidad en las distribuciones para todas las variables (sig. 0,097 a $0,798)$, nivel de significancia $\alpha 0,05$. 
Cuando fue observada la línea de tendencia en la figura 2, se observa que es posible aprobar $\mathrm{H}_{1}$, ya que a medida en que ocurre el emprendimiento de la dieta, los participantes se empeñan menos y presentan disminución en alcanzar el objetivo (pérdida de peso). Los indicadores indican los $p$-values para cada diferencia entre los períodos de evaluación.

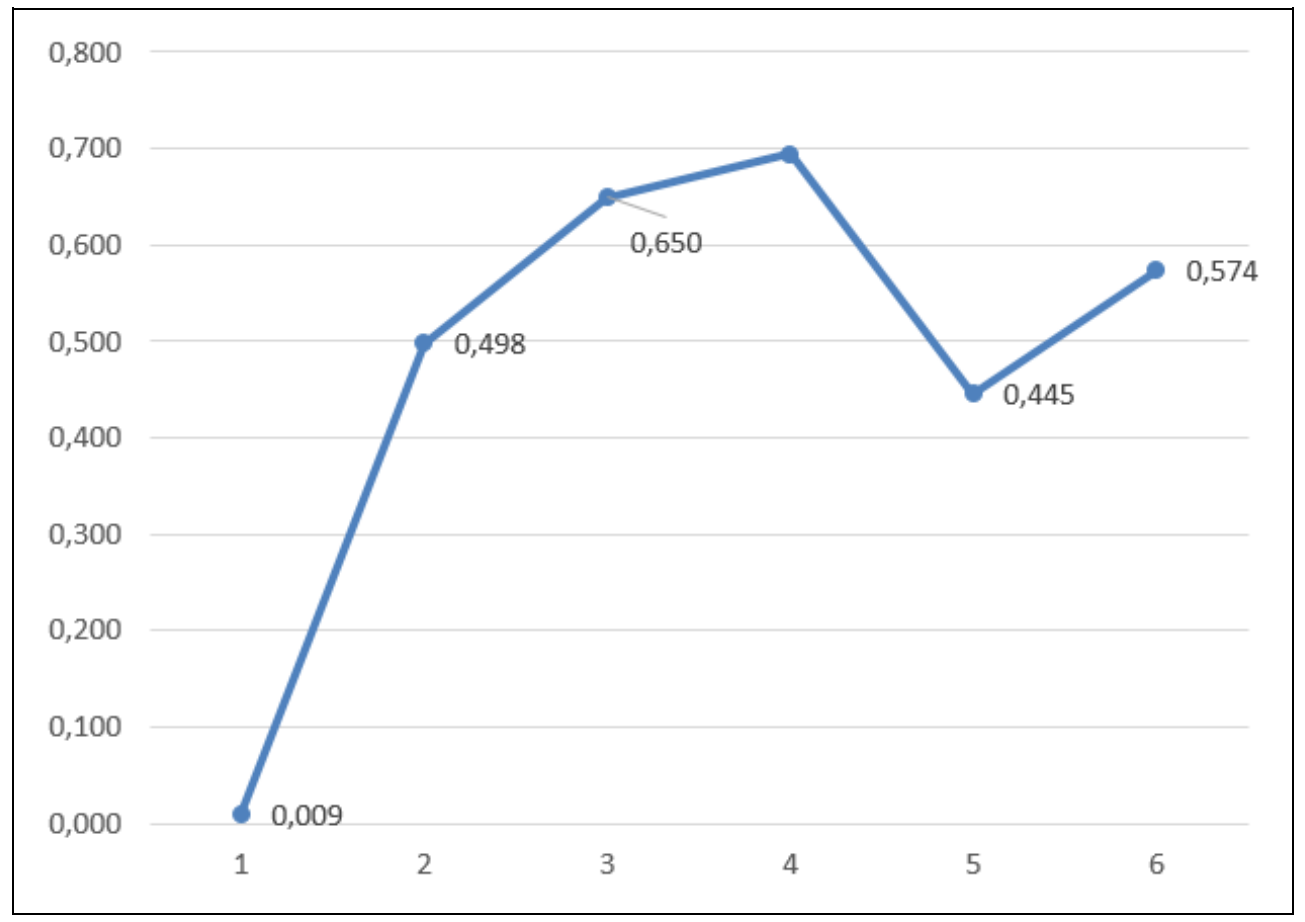

Figura 2. Serie de $p$ value para medidas de IMC

Goiânia, GO, 06 octubre 2019, Fuente: Datos del Estudio.

El inicio de la serie presenta un valor significativo para la diferencia entre los períodos $(0,009)$ de IMC, suficiente para desconsiderar $\mathrm{H}_{1}$, a medida que el emprendimiento de la dieta avanza, los valores se tornan elevados, comprobando que hay una pérdida importante a lo largo del tiempo con el esfuerzo empleado.

La primera constatación después de la relación de las variaciones intragrupos es que no hay diferencia significativa de pérdida de peso entre las 3 formas de monitoreo (adipómetro, peso y medidas).

Tabla 1. Análisis de las Diferencias entre y de entre grupos

\begin{tabular}{lcc}
\hline \hline Grupo de Investigación & $p$-value intra grupos & $p$-value entre grupos \\
\hline \hline IMC & 0,638 & 0,077 \\
PESO & 0,180 & 0,077 \\
MEDIDAS & 0,532 & 0,133 \\
CONTROL & 0,047 & \\
& Goiânia, G0, 06 octubre 2019. Fuente: Datos del Estudio
\end{tabular}


Considerando el grupo de control, que no sofrió acompañamiento por mediciones a lo largo del estudio, ocurrió significativo aumento de peso ( $p$ value 0,$047 ; 95 \%$ ).

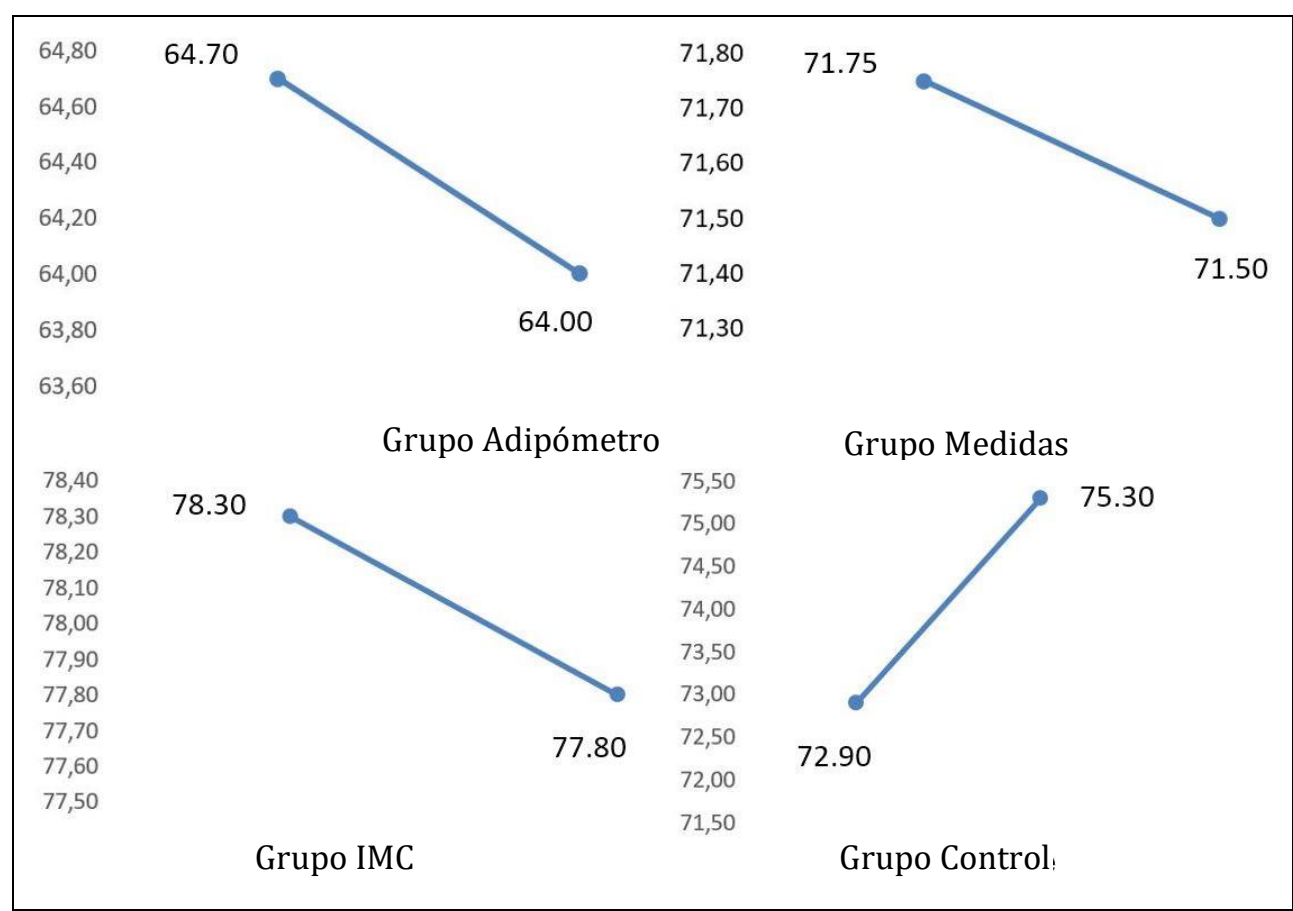

Figura 3. Análisis del Comportamiento de los Grupos

Goiânia, GO, 06 octubre 2019. Fuente: Datos del Estudio.

Los individuos al ser monitoreados, en tres grupos, registraron reducción de peso, pero no a punto de haber rechazo de la hipótesis nula (ausencia de significancia estadística). Ambas hipótesis alternativas no tuvieron sustentación, o sea, emplear técnicas distintas de monitoreo no generó efectos significativos en la práctica del autocontrol a lo largo de los 4 meses de experimento. Se destaca la cuestión que aquellos que no fueron monitoreados (control) tuvieron aumento de peso ( $p$-value 0,047 ).

En este casi experimento se investigó 3 formas de monitoreo en emprendedores de una dieta alimentar con el objetivo de verificar el autocontrol. Las hipótesis evaluadas predecían que el monitoreo en períodos fijos incrementa la taza de éxito y que la forma de controlar esas iniciativas interfiere en el resultado de la dieta. Con excepción de los emprendedores que estaban en el grupo de control, todos los demás perdieron peso.

Las pérdidas de peso no fueron significativas en los grupos "IMC", "peso" y "medidas, entretanto el aumento de peso del grupo de control puede ser considerado importante. Se concluye que la forma de monitorear un emprendimiento, como por ejemplo una dieta alimentar, no interfiere en el resultado general, pero ser monitoreado incrementa el efecto final.

Inicialmente, el hecho de no haber significancia estadística suficiente para aceptar las hipótesis alternativas sugiere una situación de atención para la casi experimentación. Dos aspectos deben ser relacionados para la cuestión: "ni toda idea funciona; cualquier científico puede atestar esa realidad" (Thaler, 2008, p. 351). Ocurre que el hecho de que los grupos 
experimentales hayan sentido reducción de peso (para participantes que no estaban dispuestos a emprender una dieta rigorosa) y que el grupo de control haya vivenciado significativo aumento de peso, sugieren algún éxito en la sustentación del autocontrol de dieta.

La analogía de prever el autocontrol con un músculo fue creada a más de dos décadas previendo dos puntos cruciales: [1] cuando el ego es llevado al agotamiento hay un corte de energía como forma de crear una reserva para otros fines; [2] así como músculos se tornan más fuertes con ejercicios, el autocontrol podría ser mejorado por esfuerzos frecuentes.

A partir de tales presupuestos, las descubiertas de la investigación apuntan para un alineamiento de la teoría sobre comportamiento, cuando se constata que el autocontrol funciona mejor al recibir ayuda externa. Existe mucha dificultad involucrada cuando se evalúa aumentos futuros en función de los sacrificios realizados en el presente.

El estudio se muestra oportuno cuando se estudia, a título de políticas públicas, adoptar prácticas de combate al sobrepeso y obesidad. Gobiernos como el brasileño planean crear acciones que contemplen rótulos para los alimentos, con destaque para super procesamientos (exceso de calorías vacías y sodio, por ejemplo); también es esperado un aumento de impuestos para los alimentos que traigan daños al consumidor (como los super endulzados). Mientras que, cabe destacar, que tales medidas deberán prevenir mudanzas comportamentales punidas como políticas públicas, tal como previsto esta pesquisa.

En esta investigación algunas limitaciones deben ser observadas, principalmente en lo que se refiere a la generalización de los resultados. Por tratarse de una muestra pequeña, la metodología debe obedecer a rigorosos procedimientos de control para variables extrañas. Se registra también una agenda de investigaciones futuras para experimentos que trabajen el autocontrol en otras situaciones distintas de una dieta. 


\section{Referências Bibliográficas}

Ariely, Dan., \& Wertenbroch, Klaus. (2002). Procrastination, deadlines, and performance: Selfcontrol by precommitment. Psychological Science, 13(3), 219-224. Acessado em 15 de setembro de 2019, de: https://doi.org/10.1111/1467-9280.00441

Baumeister, Roy F., Gailliot, Mathews., Dewall, Natan., \& Oaten, Megan. (2006). Self-Regulation and Personality: How Interventions Increase Regulatory Success, and How Depletion Moderates the Effects of Traits on Behavior. Journal of Personality, December 2006, 17741802. Acessado em 01 de setembro de 2019, de: https://doi.org/10.1111/j.14676494.2006.00428.x

Baumeister, Roy. F., Heatherton, Todd F., \& Tice, Dianne. M. (1994). Losing control: How and why people fail at self-regulation (A. Press (ed.)). Academic Press.

Beames, Joanne., Schofield, Timothy., \& Denson, Thomas. (2017). A Meta-analysis of Improving Self-control with Practice Joanne. In Centre for Mental Health, Melbourne School of Population and Global Health, The University of Melbourne.

Beedie, Crhistopher J., \& Lane, Andrew M. (2012). Personality and Social Psychology Review. Personality and Social Psychology Review, 16(2), 143-153. Acessado em 11 de setembro de 2019, de: https://doi.org/10.1177/1088868311419817

Calder, Bobby., Malthouse, Edward C., \& Maslowska, Ewa. (2016). Commentary on the Special Issue: Brand Marketing, Big Data and Social Innovation as Future of Research Directions for Engagement. Journal of Marketing Management, 32(5-6), 579-585. Acessado em 11 de setembro de 2019, de: http://perscom.socsci.uva.nl/publications/calder-b-malthouse-e-cmaslowska-e-2016-commentary-on-the-special-issue-brand-marketing-big-data-andsocial-innovation-as-future-of-research-directions-for-engagement-journal-of-marke/

Friese, Malte., Frankenbach, Julius., Job, Veronika., \& Loschelder, David D. (2017). Does SelfControl Training Improve Self-Control? A Meta-Analysis. Perspectives on Psychological Science, 12(6), 1077 -1099. Acessado em 11 de setembro de 2019, de: https://doi.org/10.1177/1745691617697076

Gailliot, Mathews T., Baumeister, Roy., Dewall, Natan., Maner, Jon., Plant, Ashby., Tice, Dianne., Brewer, Lauren., \& Schmeichel, Brandon. (2007). Self-Control Relies on Glucose as a Limited Energy Source: Willpower Is More Than a Metaphor. Journal of Personality and Social Psychology, 92(2), 325-336. Acessado em 01 de setembro de 2019, de: https://doi.org/10.1037/0022-3514.92.2.325

Gailliot, M. T., \& Baumeister, R. F. (2007). The Physiology of Willpower : Linking Blood Glucose to Self-Control. Personality and Social Psychology Review, 11(4), 303-327. Acessado em 05 de setembro de 2019, de: https://doi.org/10.1177/1088868307303030

Hagger, Martin., Chatzisarantis, Nikos., Alberts, Hugo., Anggono, Calvin., Batailler, Cedric., Birt, Angela., Brand, Raulf., Brandt, Mark., Brewer, Gene., Bruyneel, Sabrina., Calvillo, Dustin., Campbell., Keith., Cannon, Peter., Carlucci, Marianna., Carruth, Nicholas., Cheung, Tracy., Crowell., De Ridder, Denise., e cols. (2016). A Multilab Preregistered Replication of the Ego- 
Depletion Effect. Perspectives on Psychological Science, 11(4), 546-573. Acessado em 05 de Agosto de 2019, de: https://doi.org/10.1177/1745691616652873

Holton, Richard. (2009). Willing, Waiting, Wanting. Oxford University Press.

Job, Veronika., Walton, Gregory., Bernecker, Katharina., \& Dweck, Carol. (2013). Beliefs about willpower determine the impact of glucose on self-control. Psychological and Cognitve Sciences, 110(37), 14837-14842. Acessado em 05 de Agosto de 2019, de: https://doi.org/10.1073/pnas.1313475110

Job, Veronika., Walton, Ggregory., Bernecker, Katharina., \& Dweck, Carol. (2015). Implicit theories about willpower predict self-regulation and grades in everyday life. Journal of Personality and Social Psychology, 108(4), 637-647. Acessado em 05 de Agosto de 2019, de: https://doi.org/10.1037/pspp0000014

Kahneman, Daniel. (2011). Thinking, fast and slow. Macmillan.

Kotabe, Hiroki P., \& Hofmann, Wilhelm. (2015). On Integrating the Components of Self-Control. Perspectives on Psychological Science, 10(5), 618-638. Acessado em 25 de Agosto de 2019, de: https://doi.org/10.1177/1745691615593382

Laibson, David. (1996). Hyperbolic Discount Functions, Undersaving, and Savings Policy. National Bureau of Economic Research, 1-49. Acessado em 25 de Agosto de 2019, de: https://doi.org/10.3386/w5635

Laibson, David. (1997). Golden Eggs and Hyperbolic Discounting. The Quarterly Journal of Economics, 112(2), 443-477.

Mischel, Walter., Ebbsen, Ebbe., \& Zeiss, Antonette R. (1972). Cognitive and attentional mechnaisms in delay of gratification. Journal of Personality and Social Psychology, 21(2), 204-218.

Muraven, Mark., \& Baumeister, Roy F. (2000). Self-regulation and depletion of limited ressources: Does self-control remble a muscle? Psychological Bulletin, 126(2), 247-259. Acessado em 02 de Agosto de 2019, de: https://doi.org/10.1037//0033-2909.126.2.247

O’Donoghue, Ted., \& Rabin, Mathew. (1992). Doing It Now or Later. The American Economic Revew, 103-124.

O'Donoghue, Ted., \& Rabin, Mathew. (2001). Choice and procrastination* ted o'donoghue and matthew rabin. The Quarterly Journal of Economics, 116(February 2001), 121-160. Acessado em 02 de Agosto de 2019, de: https://doi.org/10.1162/003355301556365

Phelps, Edmund., \& Pollak, Robert. A. (1968). On second-best national saving and gameequilibrium growth. The Review of Economic Studies, 35(2), 185-199. Acessado em 02 de Agosto de 2019, de: https://doi.org/10.2307/2296547

Selart, Marcus., Karlsson, Niklas., \& Gärling, Tommy. (1997). Self-control and loss aversion in intertemporal choice. Journal of Socio-Economics, 26(5), 513-524. Acessado em 22 de Agosto de 2019, de: https://doi.org/10.1016/S1053-5357(97)90012-3

Shiv, Baba., \& Fedorikhin, Alexander. (2010). Heart and Mind in Conflict: The Interplay of Affect and Cognition in Consumer Decision Making. The Journal of Consumer Research, 26(3), 278292. 
Smith, Trenton G. (2004). The McDonald's equilibrium: Advertising, empty calories, and the endogenous determination of dietary preferences. Social Choice and Welfare, 23(3), 383413. Acessado em 22 de Agosto de 2019, de: https://doi.org/10.1007/s00355-003-0265-3

Smith, Trenton G. (2006). School of Economic Sciences Working Paper Series Reconciling Psychology with Economics: Obesity, Behavioral Biology, and Rational Overeating. In School of Economic Sciences (No. 2006-4).

Thaler, Richard. (2008). Misbehaving: The Making of Behavioral Economics. W.W. Norton.

Thaler, Richard., \& Sunstein, Cass. (2009). Nudge: Improving decisions about health, wealth, and happiness. In Nudge: Improving decisions about health, wealth, and happiness. Penguin Books. Acessado em 04 de Agosto de 2019, de: https://doi.org/10.1007/s10602-008-90562

Thaler, Richard., Sunstein, Cass., \& Balz, John. (2013). Choice Architecture. Human Agency and Behavioral Science, January 2012, 1-11. Acessado em 22 de Agosto de 2019, de: https://doi.org/10.13140/2.1.4195.2321

Wänke, Michaela. (2009). Social Psychology of Consumer Behavior. Psychology Press.

World Health Organization. (2017). Controlling the global obesity epidemic. WHO. Acessado em 22 de Agosto de 2019, de: https://www.who.int/nutrition/topics/obesity/en/ 